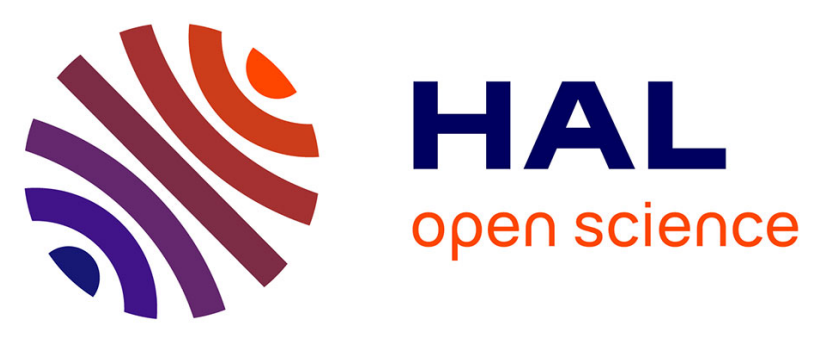

\title{
A cohesive zone element for mode I modelling of adhesives degraded by humidity and fatigue
}

M. Costa, G. Viana, Romain Créac'Hcadec, L.F.M. da Silva, R.D.S.G.

Campilho

\section{- To cite this version:}

M. Costa, G. Viana, Romain Créac'Hcadec, L.F.M. da Silva, R.D.S.G. Campilho. A cohesive zone element for mode I modelling of adhesives degraded by humidity and fatigue. International Journal of Fatigue, 2018, 112, pp.173-182. 10.1016/j.ijfatigue.2018.03.014 · hal-01783740

HAL Id: hal-01783740

https://hal-ensta-bretagne.archives-ouvertes.fr/hal-01783740

Submitted on 22 Mar 2019

HAL is a multi-disciplinary open access archive for the deposit and dissemination of scientific research documents, whether they are published or not. The documents may come from teaching and research institutions in France or abroad, or from public or private research centers.
L'archive ouverte pluridisciplinaire HAL, est destinée au dépôt et à la diffusion de documents scientifiques de niveau recherche, publiés ou non, émanant des établissements d'enseignement et de recherche français ou étrangers, des laboratoires publics ou privés. 


\title{
A cohesive zone element for mode I modelling of adhesives degraded by humidity and fatigue
}

\author{
M. Costa ${ }^{\mathrm{a}}$, G. Viana ${ }^{\mathrm{a}}$, R. Créac'hcadec ${ }^{\mathrm{b}}$, L. F. M. da Silva ${ }^{\mathrm{c}, *}$, R. D. S. G. Campilho ${ }^{\mathrm{a}, \mathrm{d}}$ \\ ${ }^{a}$ INEGI - Rua Dr Roberto Frias 400, 4200-465 Porto, Portugal \\ ${ }^{b}$ ENSTA - Bretagne, 2 rue François Verny, 29806 Brest Cedex 9, France \\ ${ }^{c}$ FEUP - Rua Dr Roberto Frias s/n, 4200-465 Porto, Portugal \\ ${ }^{d}$ ISEP - Rua Dr. António Bernardino de Almeida, 431, 4249-015 Porto, Portugal
}

\begin{abstract}
A finite element is proposed, based on the cohesive zone model approach and implemented as a user element, for the modelling of adhesively bonded joints subjected to degradation by humidity and fatigue using software ABAQUS ${ }^{\circledR}$. Functionality included in this element that is not available using standard cohesive zone elements includes: (a) various types of traction-separation laws, such as triangular with exponential softening, trapezoidal and exponential, (b) an intuitive and easy to use graphical interface built in MATLAB that helps visualize all traction-separation laws, create the mesh, run the simulation and visualize the results, (c) custom degradation laws for both humidity and fatigue which allow the user to easily model the effects of said degrading parameters. It is shown that the trapezoidal traction-separation law is the most appropriate to model the experimental data in both unaged and aged specimens. The proposed fatigue degradation approach correctly predicts the number of cycles until failure of all unaged and aged conditions, thus proving itself as a very useful tool capable of modelling a vast array of experimental conditions and details that adhesive joints are subjected to in real world applications.
\end{abstract}

Keywords: Cohesive zone model, traction-separation law, finite element analysis, humidity, fatigue, environmental degradation.

\section{Introduction}

Adhesive joints are being increasingly used in structures mainly due to their attractive strength to weight ratio, which leads to an overall reduction in weight, costs and, in the case of industries such as the automotive and aeronautical, emissions [1]. Due to the prevalence of adhesive joints in critical structures, precise experimental [2] and numerical methodologies [3] must be developed to characterise and predict joint behaviour for both static and cyclic conditions, while also evaluating the effect that environmental factors may have in a joint. Experimentally, temperature and humidity may be applied to standardized specimens such as bulk and double cantilever beams (DCBs) to obtain both static and cyclic behaviour, but numerical prediction of said phenomena is still not fully understood.

\footnotetext{
*Corresponding author. Phone number: +351 225081706

Email address: lucas@fe.up.pt (L. F. M. da Silva)
} 
Since the introduction of the cohesive zone model (CZM) concept [4 and its application to finite elements [5], adhesive joints have frequently adopted CZM to model the adhesive layer because of its ability to model delamination. CZM relates the stresses in the material with the relative displacement between adjacent nodes (by using traction-separation laws) and provides an easy to implement methodology that provides accurate results [6]. Some adhesive properties are used to build the traction-separation law (such as the tensile/shear stresses and fracture toughness), which provides limits related to what the specific material can withstand before failing. Various types of traction-separation laws exist: the simplest one being the triangular law, implemented in ABAQUS $^{\circledR}$ (2017 being the latest available version) and used in most numerical simulations, trapezoidal law, exponential law, and some more complex types of laws [7, 8]. To use these more complex CZM laws in ABAQUS $^{\circledR}$, one must go through some trouble as they are not directly implemented, thus the information of the damage variable as a function of displacement must first be created [9] (using external routines coded in, for example, MATLAB or python) and only then inserted in ABAQUS ${ }^{\circledR}$, adding some complexity to the procedure.

The presence of humidity in a joint influences the material parameters, thus to model joints degraded by humidity using CZM the traction-separation law parameters must be adapted accordingly. This is normally done manually for each degradation test, where the CZM parameters are input already affected by the level of humidity (taken from experimental tests) [10, 11], while other approaches consist in using ABAQUS ${ }^{\circledR}$ material degradation subroutines to model moisture degradation [12].

Approaches for fatigue analysis using CZM generally opt for progressively applying damage to the element using a custom formulation to mimic fatigue degradation, which is in itself based on experimental details. The vast majority of fatigue implementations using the CZM approach update the damage variable as a function of several fatigue cycles for each iteration, controlling the number of cycles evaluated by each numerical jump: in [13], the fatigue damage parameter of the CZM is defined as a function of a certain range of fatigue cycles controlled by a pre-defined maximum variation, geometric properties and experimental Paris Law data for mode I; [14] controls the damage parameter as a function of the range of applied strain energy release rate and is applicable to mode I, mode II and mixed-mode conditions; [15] opts for a similar approach of controlling the fatigue damage parameter as a function of a range of considered fatigue cycles and some material constants that should be calibrated against experimental tests; [16] takes a similar approach to the previous works and defines the variation of the damage parameter as a function of the damaged area and the corresponding crack growth rate which must be determined experimentally for different loading conditions. Other works opt for a cycle-by-cycle approach: in [17, the stiffness is controlled as a function of the initial stiffness (without fatigue damage) and a inverse exponential formula that takes into account the cycle being simulated and the CZM opening and final displacements; while [18] defines the fatigue damage parameter of the CZM as a function of the Heaviside function for gradual fatigue degradation. The cycle-by-cycle approaches turn out to be very computationally intensive and not beneficial in terms of results, as approaches based on considering various cycles in each numerical jump yield satisfactory results faster. Generally, all approaches for modelling fatigue 
follow some kind of complex mathematical model, while works that are based on relationships derived from experimental data are scarce.

The objective of this work is to provide a complete finite element based on the CZM approach that corrects various of the previously mentioned problems. By incorporating several types of traction-separation laws such as trapezoidal and exponential, the need for complex workarounds to determine the tabular representation of the CZM is eliminated. Modelling of joints degraded with humidity is also integrated in the element by using a general law for degradation of the properties as a function of the humidity content, thus avoiding manual tweaking of the CZM parameters to fit a specific humidity concentration. Finally, modelling fatigue is also taken into account, by using relationships that are obtained using experimental data, thus guaranteeing reproducibility of the results. The proposed finite element is based on the cohesive zone model approach and coded in FORTRAN for use in ABAQUS ${ }^{\circledR}$, with the final aim of providing results by using formulations for element degradation. This paper is structured in the following way: section 2 presents everything related to the formulation, from the base finite element code to the equations implemented in each CZM law and degradation behaviours for humidity and fatigue, while section 3 focuses on the MATLAB developed interface, followed by section 4 which compares experimental data with numerical results while discussing all results, and finally the conclusions are drawn.

\section{Formulation}

\subsection{Finite element method}

The finite element method (FEM) summarily consists in studying a domain of arbitrary complexity by discretizing it in several sub-domains called elements, calculating the displacement of each element as a function of the applied loads/boundary conditions, and then combining the information from all elements into the whole model. Mathematically, FEM consists in solving equation:

$$
[k] \cdot\{d\}=\{f\}
$$

where $[k]$ is the stiffness matrix (constructed as a function of the material parameters), $\{d\}$ is the displacements vector (to be determined by FEM) and $\{f\}$ is the external forces vector (where the applied forces are considered). The cohesive zone model (CZM) approach, used in delamination problems, is based on traction-separation laws, which correlate the stress existing in a specific point of the bond as a function of the displacement that point is subjected to. More information of the derivation of $[k]$ and $\{f\}$ for general FEM problems may be found in the literature [19], but for the specific case of using CZM in FEM, $[k]$ and $\{f\}$ from Equation 1 may be defined as:

$$
\begin{aligned}
{[k] } & =w \cdot[B]^{T} \cdot\left[T_{d}\right] \cdot[B] \\
\{f\} & =w \cdot[B]^{T} \cdot\{T\}
\end{aligned}
$$

where $w$ is the width of the element, $[B]$ is the global displacement-separation relation matrix (defined further ahead), and both $\{T\}$ and $\left[T_{d}\right]$ are a vector and matrix, respectively, in which the traction-separation 
law considerations are included, such that each traction-separation law type (see Figure 1) will have different $\{T\}$ and $\left[T_{d}\right]$ formulations.

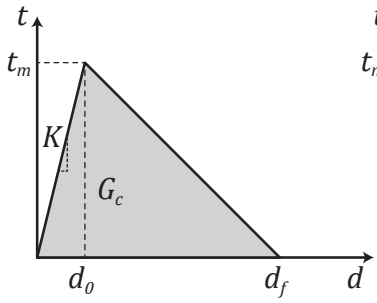

(a)

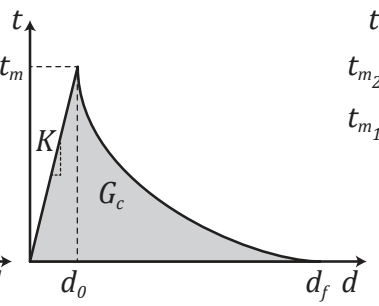

(b)

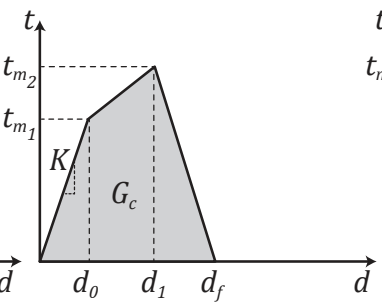

(c)

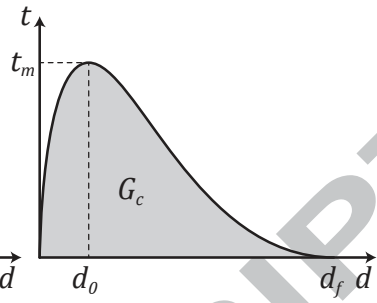

(d)

Figure 1: Various traction-separation laws: (a) triangular, (b) triangular with non-linear softening, (c) trapezoidal with nonconstant plateau, (d) exponential.

$\{T\}$ and $\left[T_{d}\right]$ may be defined, for mode I loadings in the local coordinate system (see Figure 22), as:

$$
\{T\}=\left\{\begin{array}{c}
0 \\
t(d)
\end{array}\right\} \quad\left[T_{d}\right]=\left[\begin{array}{cc}
0 & 0 \\
0 & t^{\prime}(d)
\end{array}\right]
$$

where $t(d)$ corresponds to the equation that defines the CZM law under consideration, see Figure 1 Thus defining $\{T\}$ and $\left[T_{d}\right]$ allows for modelling any desired CZM shape. Various types of curves can be considered, as seen in Figure 1, and different types of relationships are usually associated with different situations and selected as a function of either geometry or adhesive properties. For example, the simple triangular shape is often used with brittle adhesives, as the results are usually very satisfactory for these situations, while the trapezoidal shape is recommended when studying ductile adhesives due to the added plateau of the curve which helps modelling the ductile fracture of those types of adhesives.

\subsection{Cohesive element}

Basic FEM concepts such as number of nodes, shape functions and matrices must be defined for the developed cohesive element. Figure 2 shows a portion of a DCB specimen with the location where the proposed element should be used, together with a representation of the element: the DCB will be loaded to the left of the represented portion, inducing separation of the nodes connected through the cohesive element, inducing material degradation and, ultimately, crack propagation when fully damaged (starting at the edge nearer to the application of the load).

Figure 2 shows the element in two different coordinate systems: global, which are the original coordinates of each node (related to the rest of the model), and local, which is a coordinate system specifically for the element. An element with 4 nodes was chosen, and in standard 4 noded elements 4 shape functions should be chosen, which interpolate the solution between the discrete values obtained at the mesh nodes (more information may be found in the literature [19]). For formulation purposes, the height of the cohesive element is considered null $\left(e l_{y}=0 \mathrm{~mm}\right)$, thus only shape functions for nodes $1 / 4$ and $2 / 3$ are needed, as the 

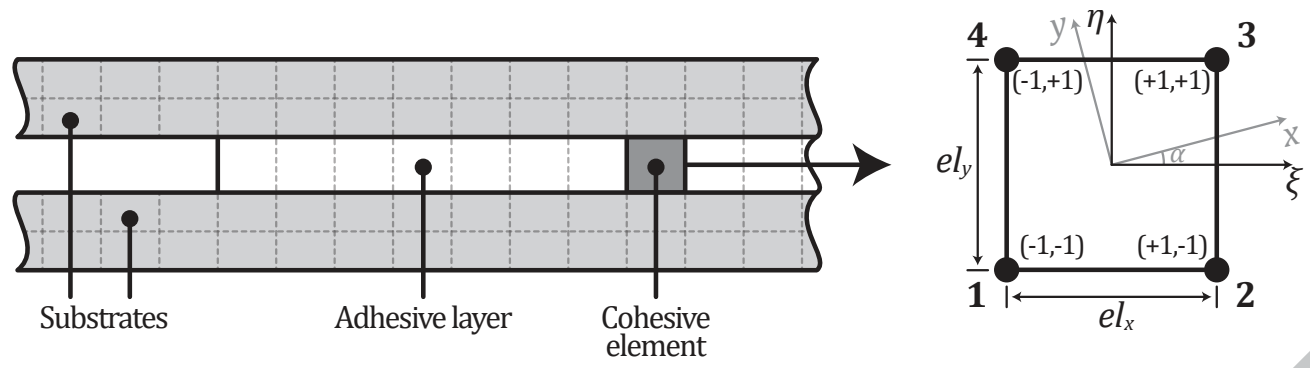

Figure 2: Section of a discretized DCB specimen (exaggerated height of the adhesive layer for easier visualization) and detail of the cohesive zone element in both global $(x, y)$ and local $(\xi, \eta)$ coordinates.

same function applies to both nodes at a specific $\xi$ coordinate:

$$
N_{1,4}=\frac{1}{2}(1-\xi) \quad ; \quad N_{2,3}=\frac{1}{2}(1+\xi)
$$

For application purposes, the cohesive element may serve as a contact element $\left(e l_{y}=0 \mathrm{~mm}\right)$ or as an element with finite height $\left(e l_{y}>0 \mathrm{~mm}\right)$, where in the latter case the displacement calculations related to the cohesive zone model assume the initial element height as the starting point. It should be noted that 4 nodes were selected as a starting step and the element may be further improved to support additional nodes (such as 6 or 8 nodes), which will help with big models with a high number of total elements (reducing the number of cohesive elements will increase the speed of the simulation and reduce computational time).

Matrix $[N]$ can now be built, which represents the matrix of the shape functions, using the information from Equation 4 such that:

$$
[N]=\left[\begin{array}{cccccccc}
N_{1,4} & 0 & N_{2,3} & 0 & N_{2,3} & 0 & N_{1,4} & 0 \\
0 & N_{1,4} & 0 & N_{2,3} & 0 & N_{2,3} & 0 & N_{1,4}
\end{array}\right]
$$

And matrix $[B]$, which represents the strain-displacement matrix, is defined by:

$$
[B]=[R][N]=\left[\begin{array}{cc}
\cos (\alpha) & \sin (\alpha) \\
-\sin (\alpha) & \cos (\alpha)
\end{array}\right]\left[\begin{array}{cccccccc}
N_{1,4} & 0 & N_{2,3} & 0 & N_{2,3} & 0 & N_{1,4} & 0 \\
0 & N_{1,4} & 0 & N_{2,3} & 0 & N_{2,3} & 0 & N_{1,4}
\end{array}\right]
$$

Where $[R]$ represents the transformation matrix from global to local coordinates, and $\alpha$ is the angle between said coordinate systems (see Figure2). With this matrix defined, all information needed to calculate Equation 2 is obtained as well as a fully working finite element code.

\subsection{Traction-separation laws}

\subsubsection{Pre-defined types of traction-separation laws}

As mentioned above, defining $\{T\}$ and $\left[T_{d}\right]$ (Equation 3 ) is dependent on the formulation of each type of traction-separation law. Table 1 summarizes how $t_{i}(d), t_{i}^{\prime}(d)$ and $G_{i}$ are defined for each zone $i$ of each CZM, i.e. the number of distinct separations dictated by differing behaviour inside the same curve (for example a change from linear function to exponential), thus both the triangular CZMs have 2 zones $\left(0 \rightarrow d_{0}\right.$ and 
$\left.d_{0} \rightarrow d_{f}\right)$, trapezoidal has 3 zones $\left(0 \rightarrow d_{0}, d_{0} \rightarrow d_{1}\right.$ and $\left.d_{1} \rightarrow d_{f}\right)$ and finally the exponential CZM has 1 zone $\left(0 \rightarrow d_{f}\right)$ because the whole curve is defined by a single equation (Figure 1).

Table 1: Equations needed to define each zone of each implemented traction-separation law.

\begin{tabular}{|c|c|c|c|c|}
\hline CZM law & $\begin{array}{l}\text { Number } \\
\text { of zones }\end{array}$ & Traction & Derivative & Area \\
\hline Triangular & 2 & $\begin{array}{l}t_{1}(d)=\frac{t_{m} d}{d_{0}} \\
t_{2}(d)=t_{m}\left(1-\frac{d-d_{0}}{d_{f}-d_{0}}\right)\end{array}$ & $\begin{array}{l}t_{1}^{\prime}(d)=\frac{t_{m}}{d_{0}} \\
t_{2}^{\prime}(d)=\frac{-t_{m}}{d_{f}-d_{0}}\end{array}$ & $\begin{aligned} G_{1} & =\frac{t_{m} d_{0}}{2} \\
G_{2} & =\frac{t_{m}\left(d_{f}-d_{0}\right)}{2}\end{aligned}$ \\
\hline $\begin{array}{l}\text { Triangular } \\
\text { (non-linear } \\
\text { softening) }\end{array}$ & 2 & $\begin{array}{l}t_{1}(d)=\frac{t_{m} d}{d_{0}} \\
t_{2}(d)=t_{m}\left(1-\frac{d-d_{0}}{d_{f}-d_{0}}\right)^{C}\end{array}$ & $\begin{array}{l}t_{1}^{\prime}(d)=\frac{t_{m}}{d_{0}} \\
t_{2}^{\prime}(d)=t_{m} C \frac{\left(\frac{-d_{f}+d}{-d_{f}+d_{0}}\right)^{C}}{-d_{f}+d}\end{array}$ & $\begin{array}{l}G_{1}=\frac{t_{m} d_{0}}{2} \\
G_{2}=\frac{t_{m}\left(d_{f}-d_{0}\right)}{K+1}\end{array}$ \\
\hline Trapezoidal & 3 & $\begin{array}{l}t_{1}(d)=\frac{t_{m_{1}} d}{d_{0}} \\
t_{2}(d)=t_{m_{1}}+\left(t_{m_{2}}-t_{m_{1}}\right) \frac{d-d_{0}}{d_{1}-d_{0}} \\
t_{3}(d)=t_{m_{2}} \frac{d_{f}-d}{d_{f}-d_{1}}\end{array}$ & $\begin{aligned} t_{1}^{\prime}(d) & =\frac{t_{m_{1}}}{d_{0}} \\
t_{2}^{\prime}(d) & =\frac{t_{m_{2}}-t_{m_{1}}}{d_{1}-d_{0}} \\
t_{3}^{\prime}(d) & =\frac{-t_{m_{2}}}{d_{f}-d_{1}}\end{aligned}$ & $\begin{aligned} G_{1} & =\frac{t_{m} d_{0}}{2} \\
G_{2} & =-\frac{\left(t_{m_{1}}+t_{m_{2}}\right)\left(d_{0}-d_{1}\right)}{2} \\
G_{3} & =\frac{t_{m_{2}}\left(d_{f}-d_{1}\right)}{2}\end{aligned}$ \\
\hline Exponential & 1 & $t_{1}(d)=d \frac{t_{m}}{d_{0}} e^{1-\frac{d}{d_{0}}}$ & $t_{1}^{\prime}(d)=-\left(\frac{d}{d_{0}}-1\right) \frac{t_{m}}{d_{0}} e^{1-\frac{d}{d_{0}}}$ & $G_{1}=d_{0} t_{m} e$ \\
\hline
\end{tabular}

In Table 1, all laws, with the exception of exponential, have an initial stiffness value $(K$ in Figure 1$)$ defined as $K=E / h_{a}$, where $E$ is the Young's modulus of the adhesive and $h_{a}$ is the thickness of the adhesive layer. This value is necessary to define $d_{0}$ in those curves, otherwise that would not be possible. Furthermore, the adhesive fracture toughness, $G_{c}$, is the sum of all the areas of each curve, such that $G_{c}=\sum G_{i}$. Also, all traction and fracture values are given at the start of a simulation (based on adhesive properties), thus what remains to be defined are the specific displacements of each curve (i.e. $d_{0} / d_{1} / d_{f}$ ), which can be obtained by rearranging the equations for the area of the curves. Regarding the triangular law with non-linear softening, $C$ represents the exponent of the softening zone, such that $0<C<\infty$.

\subsubsection{Custom traction-separation law}

The previously shown shapes are useful to model a variety of situations, but there may be a need to implement more complex types of traction-separation laws for specific problems. The proposed user element 20] formulation takes this behaviour into account by evaluating fully custom traction-separation laws, which are created by defining the various points and also the behaviour connecting adjacent points, as shown in Figure 3 .

It works by defining an equation between each two consecutive points (as a function of their coordinates and line evolution - linear, quadratic, cubic, etc.), such that the equations can then be probed in the finite element code for the specific stress value for the displacement. The advantage of this feature is that there are infinite possibilities for traction-separation curves, allowing the user to adapt the CZM in a way that fully fits its experimental data. Further advantages may include different types of custom CZM curves for each mode that allows to better capture the behaviour of a specific mode, and also the fact that a traction-separation 

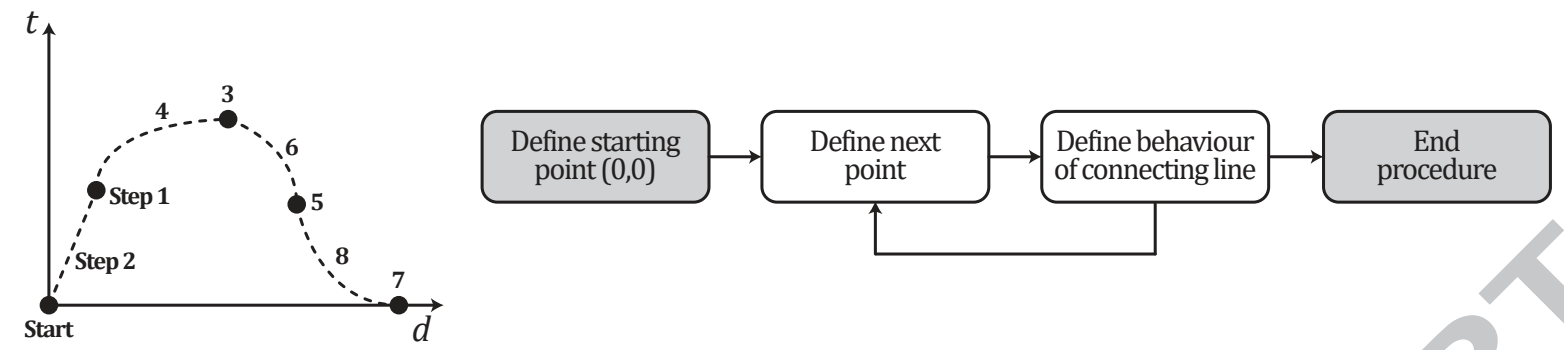

Figure 3: Example of a custom traction-separation law with each step needed for its full definition (left), flowchart of the procedure (right).

law may be derived from an experimental curve and evaluated in the cohesive zone element directly (by using techniques such as the J-integral [21]).

\subsection{Degradation by humidity}

The presence of humidity in an adhesive translates into a degradation of its mechanical properties [22, 23. 24], and the way such degradation affects specific adhesives must be understood, specifically for properties that are necessary for the definition of the CZM law (such as Young's modulus, tensile strength and fracture toughness). The traction-separation law parameters are thus adapted in the beginning of the simulation as represented in Figure 4
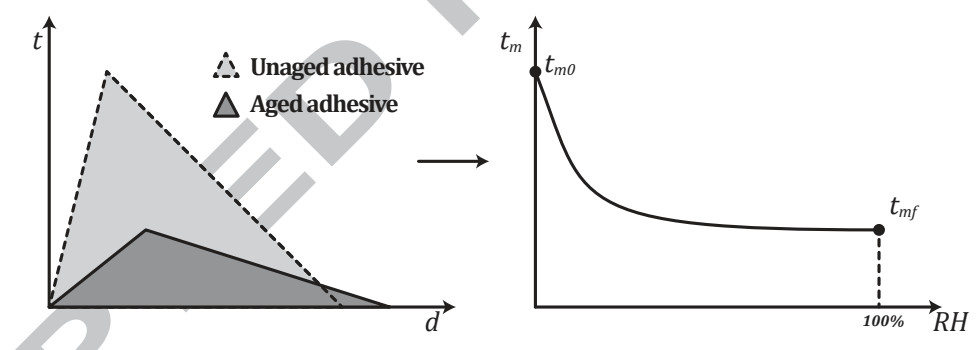

Figure 4: How the CZM is adapted as a function of the degraded adhesive parameters (left), which evolve as a function of relative humidity (right).

Experimental work is therefore needed to characterize how the mechanical properties of the adhesive itself decrease with different humidity levels, and relationships should be found on how each property decreases with an increase in humidity. Previous work has been accomplished with such objective, and the degradation of two distinct epoxy adhesives has been studied [23, which has resulted in equations that allow the CZM code to determine the mechanical properties at any level of humidity content using the following equation:

$$
y(x)=\left(y_{0}-y_{f}\right) \cdot e^{-x / C}+y_{f} \quad \text { with } \quad C=\left(10^{13} / 2\right) \cdot D \cdot m_{\infty}
$$

where $y$ is the tensile property to be degraded (Young's modulus, tensile stress or strain at failure), subscripts 0 and $f$ are, respectively, the initial and final values for that property, $x$ is the level of relative humidity present in the joint (such that $0 \leq x \leq 1$ ), and $D$ and $m_{\infty}$ are parameters for the Fick's law of 
each adhesive. Equation 7 is plotted in Figure 4 (right) for the case of tensile stress (other properties follow a similar evolution), and more details on this formulation may be found in the literature [23]. Thus, by simply providing the base mechanical properties, Fick's law parameters and relative humidity content, the cohesive element may estimate the value of each property for the specific humidity concentration and adapt the traction-separation law automatically. The presented relationship is valid for the studied cases, where a fit between Equation 7 and the experimental data was optimal.

\subsection{Degradation by fatigue}

Fatigue is characterized by a cyclic accumulation of damage in the adhesive, for loading conditions below those which would result in failure of the joint under study. This translates into a need for the cohesive element to damage the material, in a manner consistent with experimental results, through the use of degradation coefficients for the traction-separation law as a function of the applied fatigue cycles. Thus, the following degradation relationship was implemented:

$$
y(N)=y_{0}\left(1-\frac{N}{N_{f}}\right)^{K}
$$

where $y$ is the property of the cohesive zone law being degraded (such as $t_{m}$ and $G_{c}$, see Figure 1), $N$ is the cycle (or cycle range) being evaluated, $y_{0}$ is the undegraded value of that property (for $N=0$ ), $N_{f}$ is the number of cycles at failure, and $K$ is a coefficient manually fitted to experimental data which controls the degradation rate. How Equation 8 translates into pratice may be visualized in Figure 5

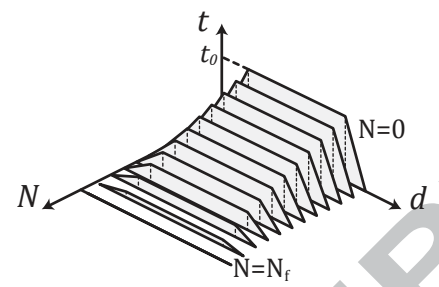

(a)

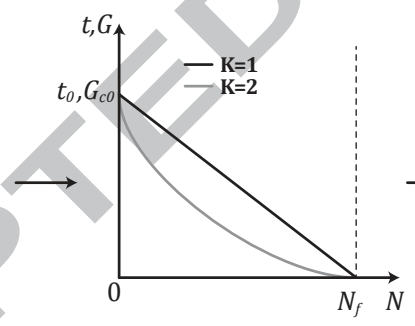

(b)

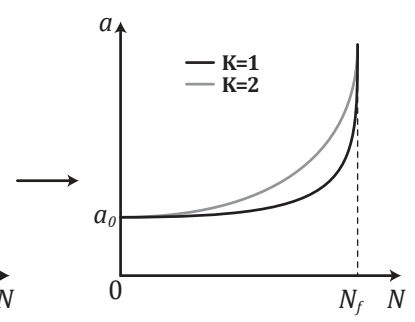

(c)

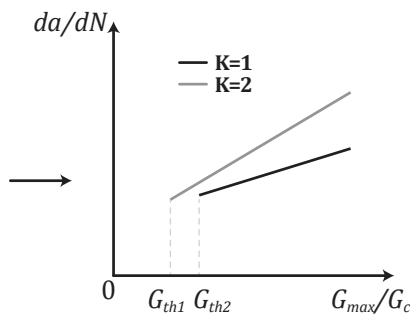

(d)

Figure 5: (a) degradation of a trapezoidal traction-separation law as a function of fatigue cycles, (b) CZM parameters change as a function of the number of cycles for two different degradation coefficients, (c) resulting crack length propagation difference, (d) corresponding fatigue crack growth curves.

The proposed methodology induces degradation such that the crack propagates until failure, but the number of cycles to failure $\left(N_{f}\right)$ must also be defined. To accomplish that, the following relationship is implemented:

$$
N_{f}=\frac{\Delta a}{(d a / d N)_{a}}
$$

where $\Delta a$ is the total length of the bonded area, and $(d a / d N)_{a}$ is an average value deduced from the Paris Law of the adhesive under study. 


\section{MATLAB interface}

Due to the complexity of the development phase (the need to test several geometries with various mesh configurations for various adhesive properties under both static and dynamic conditions), an interactive MATLAB interface ${ }^{1}$ was developed with the aim of centralizing all repetitive tasks, from creating all simulation files (ABAQUS input and FORTRAN code) to extracting the simulation results from the ABAQUS output file and display them to the user, as seen in Figure 6.

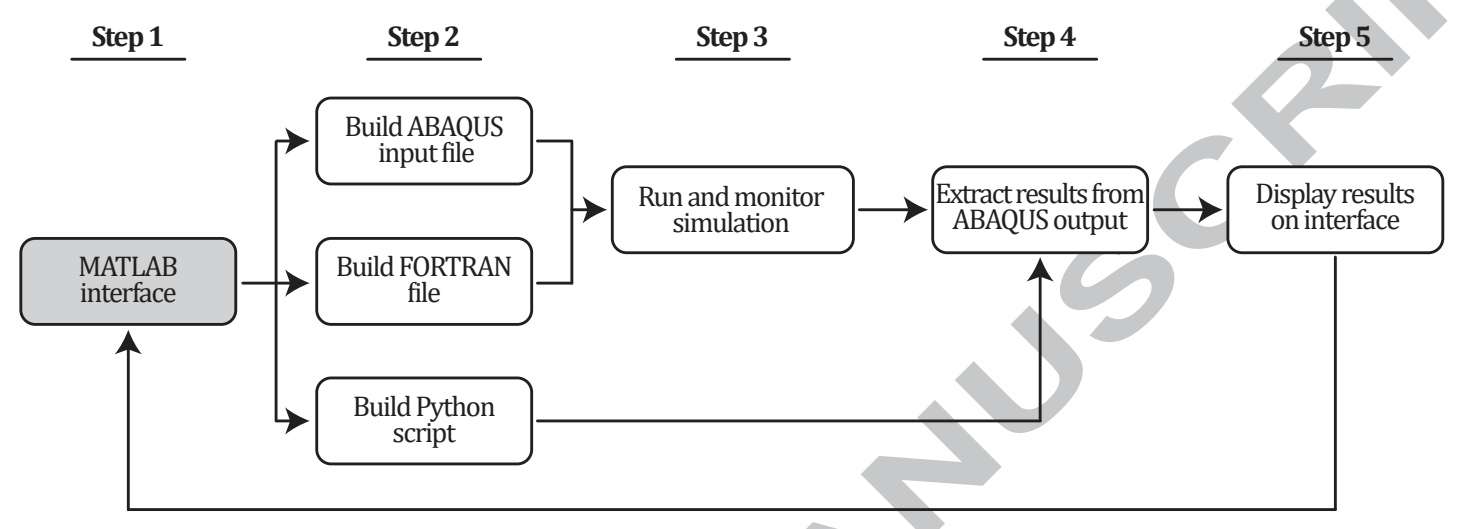

Figure 6: Flowchart of the MATLAB interface.

The advantages of having such an interface are:

- Not having to recreate an ABAQUS ${ }^{\circledR}$ model every time there is a change to a geometric/mesh parameter;

- Easily compare results from different simulations (result from each simulation is compared against experimental and previous numerical curves);

- Overlay all traction-separation laws and study the effect that changing each parameter has on the law and on the simulation result;

- Aggregate all functions needed to run the desired ABAQUS ${ }^{\circledR}$ simulation without having to open the ABAQUS ${ }^{\circledR}$ interface (otherwise needed to both build the model and visualise the results).

While the interface was initially built specifically for the development phase (i.e. it would be discarded once the cohesive element was finished), it quickly became apparent that it would also be very useful for the end user to build models and run simulations quickly and easily. The interface is divided in 5 distinct zones, as represented in Figure 7.

The first zone is related to the geometry of the specimen under study, where the various geometrical parameters are defined and are the same as those presented in Figure 8 such that two types of specimens are currently possible to test, with other types of joints possible to implement if needed.

\footnotetext{
${ }^{1}$ Available at http://www.fe.up.pt/ marcelocosta
} 


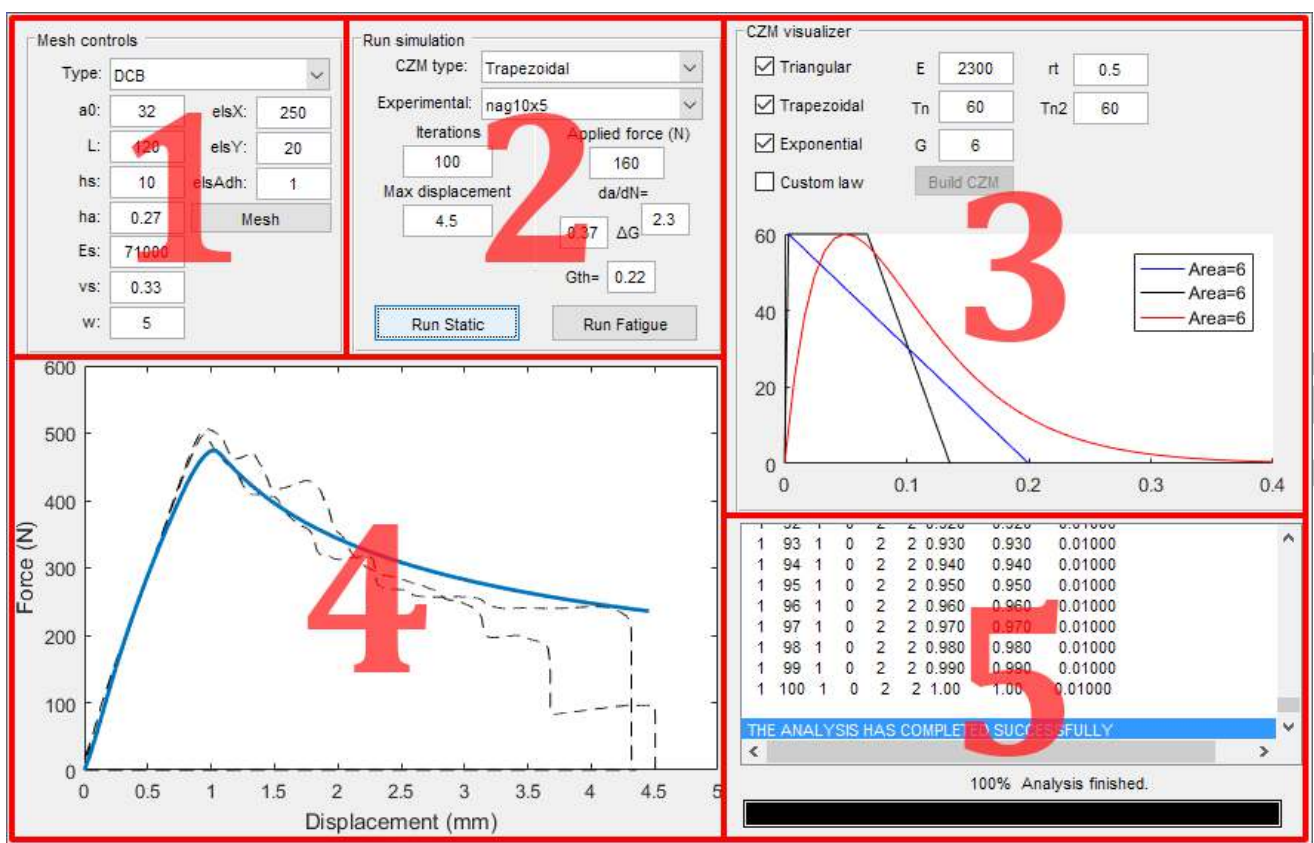

Figure 7: MATLAB interface.
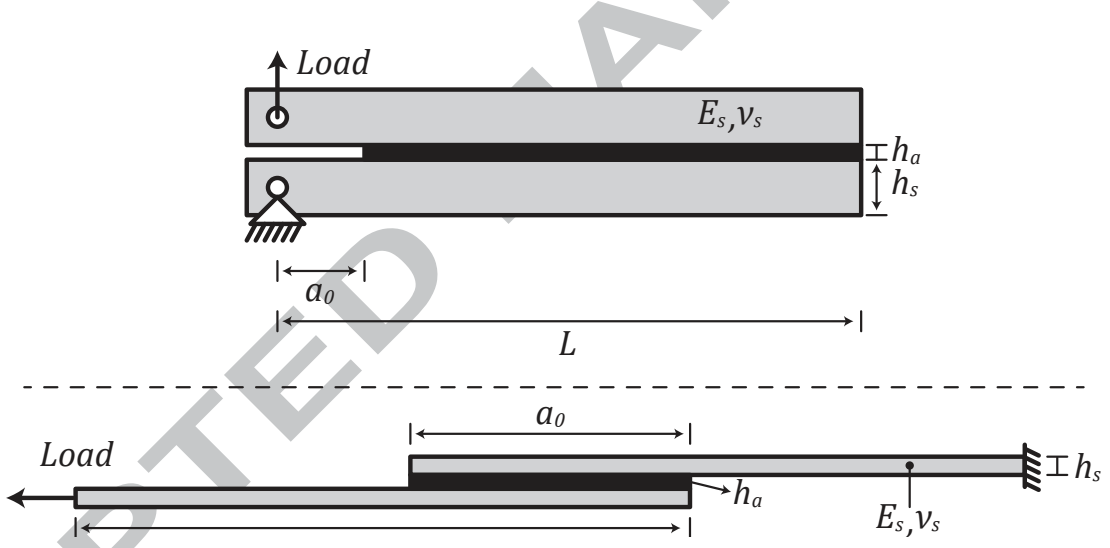

Figure 8: Relationship between physical parameters and interface variables for a Double Cantilever Beam (top), and a Single Lap Joint (bottom)

The second zone is used to start simulations and also define the simulation parameters such as maximum displacement (for static conditions) and applied load and Paris Law parameters (for fatigue conditions). Furthermore it defines the type of traction-separation law that should be evaluated, and can also load experimental curves which are used to compare against numerical results.

Zone three has two main purposes: defining the material properties (such as Young's modulus E, tensile strength $t_{m}$ and fracture toughness $G$ ), and visualizing the various types of traction-separation laws. Another feature related to this section is the definition of a custom traction-separation law, as described in Section 2.3 .2 , which is done using the interface shown in Figure 9 .

Zone four has two purposes: display the mesh and geometry prior to the start of the simulation for 


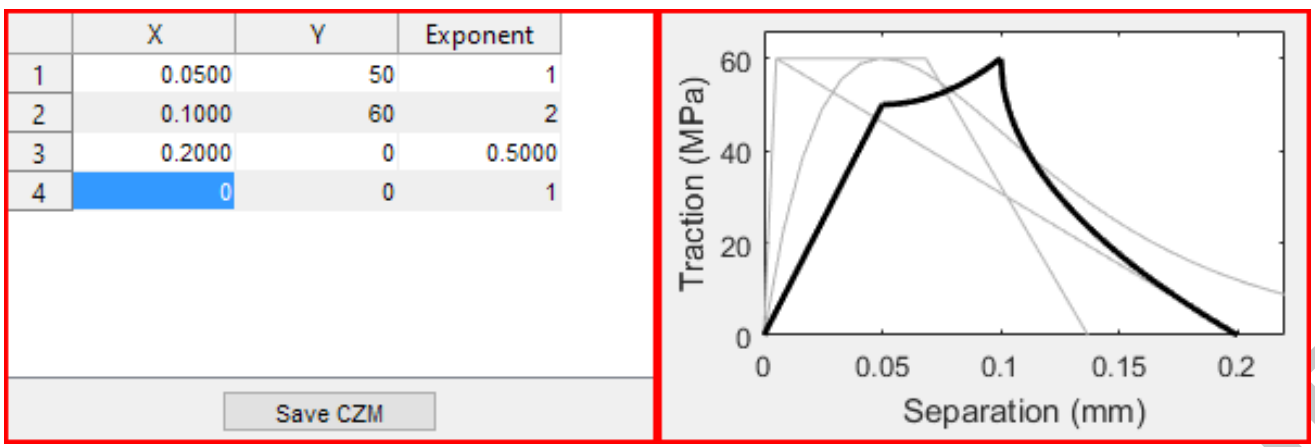

Figure 9: MATLAB interface for defining a custom traction-separation law: defining each point and type of curve connecting adjacent points (left), and resulting CZM (right).

validation purposes, and displaying the results of the simulation after the end (and, in case there is an experimental file selected in zone two, overlay the numerical results on the experimental curves).

Finally, zone five is a debugging area, showing the progress of the simulation in a progress bar while simultaneously showing the output from ABAQUS ${ }^{\circledR}$ during the analysis.

\section{Results and discussion}

The validation phase of the developed cohesive element was performed based on extensive experimental tests, with the objective of feeding enough data into the model so that the degradation laws could be obtained. Two adhesives were studied, Nagase XNR 6852-1 and SikaPower 4720, both epoxy adhesives with very different mechanical properties, as shown in Table 2 .

Table 2: Various properties of both studied adhesives [23, 25].

\begin{tabular}{|c|c|c|c|}
\hline & Property & Nagase XNR 6852-1 & SikaPower 4720 \\
\hline $\begin{array}{l}\text { Diffusion } \\
\text { properties }\end{array}$ & $\begin{array}{l}\text { Diffusion coefficient }\left(\mathrm{m}^{2} / \mathrm{s}\right) \\
\text { Mass at saturation }(\%)\end{array}$ & $\begin{array}{c}5.02 \cdot 10^{-13} \\
1.2\end{array}$ & $\begin{array}{c}0.89 \cdot 10^{-13} \\
32.5\end{array}$ \\
\hline $\begin{array}{l}\text { Mechanical } \\
\text { properties }\end{array}$ & $\begin{array}{l}\text { Young's modulus }(\mathrm{MPa}) \\
\text { Tensile strength }(\mathrm{MPa}) \\
\text { Mode I fracture toughness }(\mathrm{N} / \mathrm{mm})\end{array}$ & $\begin{array}{c}2300 \\
60 \\
6\end{array}$ & $\begin{array}{c}2100 \\
30 \\
2\end{array}$ \\
\hline $\begin{array}{l}\text { Fatigue } \\
\text { crack growth } \\
\text { properties }\end{array}$ & $\begin{array}{l}\text { Intersection } C(\mathrm{~mm} / \text { cycle }) \\
\text { Slope } m \\
\text { Crack initiation threshold } G_{t h} / G_{I c}\end{array}$ & $\begin{array}{l}0.10 \\
1.18 \\
0.55\end{array}$ & $\begin{array}{l}0.14 \\
2.18 \\
0.22\end{array}$ \\
\hline
\end{tabular}

The diffusion properties were determined by defining Fick's law, the mechanical properties using bulk tensile specimens, and the fatigue crack growth properties by determining the Paris Law for each adhesive in mode I. Further details on all methodology may be found in the literature [23, 25]. 


\subsection{Convergence tests}

The size of the mesh is an important parameter in any FEM simulation, where a balance between a coarse mesh (which will lead to faster but more inaccurate results) and a refined mesh (which will take more computational time to solve but yields more accurate results) must be found. To that end, various levels of mesh refinement were tested using the proposed element formulation, and the results may be seen in Figure 10
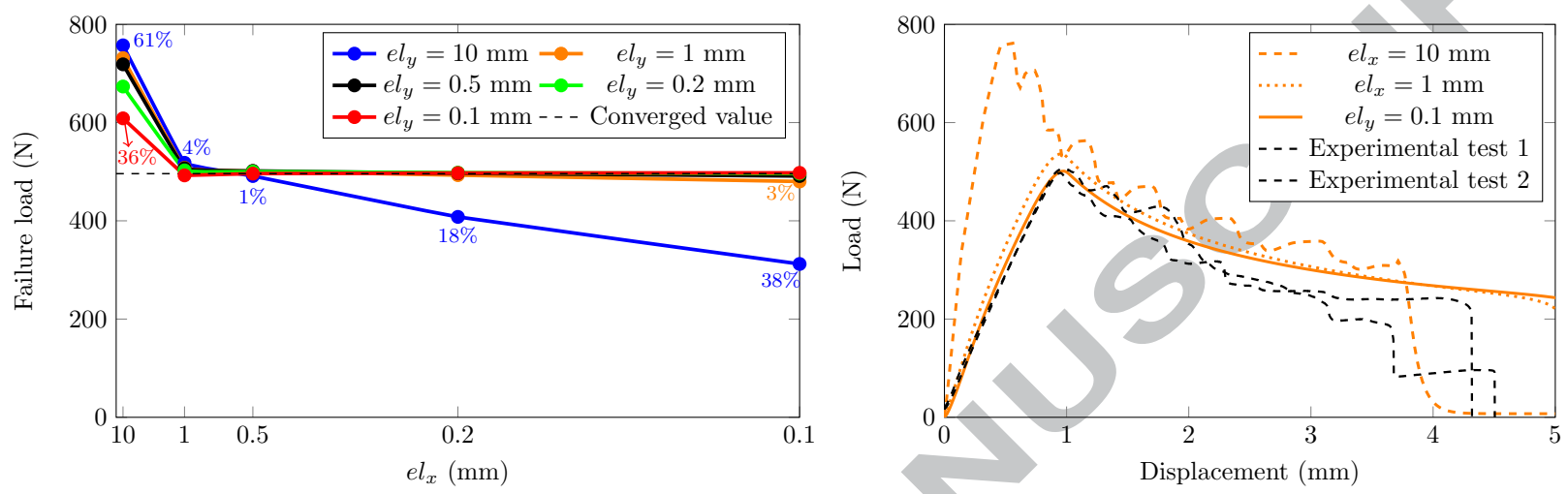

Figure 10: Effect of element size for both $x$ and $y$ direction in the simulated failure load of DCB specimens (left), and effect of element size in the $x$ direction $\left(e l_{y}=1 \mathrm{~mm}\right.$ ) on the load displacement curve (right).

The test was performed for the case of the Nagase XNR 6852-1 double cantilever beam model, and the results shown were obtained using the trapezoidal law, although the same trend is obtainable for the remaining CZM laws (in terms of reduction of the error as the element size is reduced). The dimensions of the element are $e l_{x}$ and $e l_{y}$ for the $x$ and $y$ direction, respectively, and higher mesh refinement levels yields more elements in one direction, thus a smaller dimension. $e l_{x}$ affects the entire model (both substrates and adhesive layer), while $e l_{y}$ only affects the substrates (because due to its reduced thickness, the adhesive is modelled using a single element along its height). It is visible (Figure 10 (left)) that, for almost all cases, as the number of elements in any direction increases, the failure load tends to a stable/converged value, as would be expected. The exception is the case of one single element for the substrate ( $e l_{y}=h_{s}=10 \mathrm{~mm}$ ), which even with a higher number of elements along the $x$-axis does not converge. Because of that, $e l_{y}=1 \mathrm{~mm}$ was chosen as the quickest mesh which provides good results for the comparison on Figure 10 (left), which shows that for the case of $e l_{x}=1 \mathrm{~mm}$ a curve very close to the experimental one may already be achieved. This means that considerably accurate results may be obtained using element sizes as high as $e l_{x}=e l_{y}=1 \mathrm{~mm}$, which translates to relatively fast simulation times.

\subsection{Static testing}

The geometric and material properties of the specimens (Figure 8) used in the experimental tests [23] is: $a_{0}=20 \mathrm{~mm}, L=120 \mathrm{~mm}, h_{a}=0.2 \mathrm{~mm}$ and $E_{s}=71 \mathrm{GPa}$, while $h_{s}=10 \mathrm{~mm}$ for the specimens used for Nagase XNR 6852-1 and $h_{s}=5 \mathrm{~mm}$ for the SikaPower 4720 specimens, which were selected as a function 
of adhesive toughness (higher toughness means higher stresses on the substrate, meaning the thickness must be increased to guarantee no plasticity in the substrates). The study and selection of substrate thickness is published in the same work [23] and in a previous work [26] devoted to developing reduced specimens for use in environmental degradation studies, needed to reduce the time until moisture saturation is obtained.

For the unaged condition (no relative humidity present on the joint), Figure 11 presents a comparison between experimental results for each adhesive together with numerical curves for each type of tractionseparation law implemented in the element code. The analysis was done in $2 \mathrm{D}$ using the element size $e l_{x}=e l_{y}=0.1 \mathrm{~mm}$, shown in the mesh convergence section to provide a high level of accuracy. A 3D analysis was not performed as various studies have shown that $2 \mathrm{D}$ analyses give accurate enough results for the studied geometry [3].

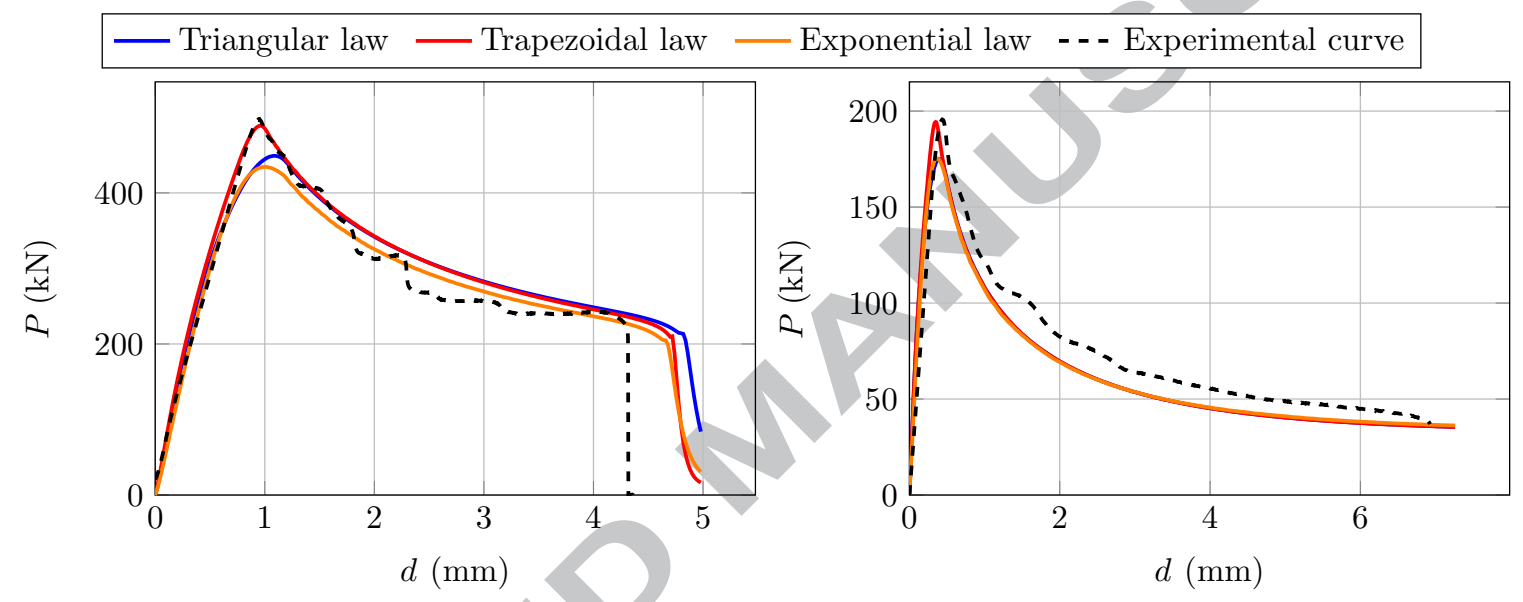

Figure 11: Experimental and numerical results for each type of traction-separation law for the two studied adhesives: Nagase XNR 6852-1 (left), SikaPower 4720 (right).

It can be seen that the numerical results fit the experimental curves, specially for the case of the Nagase XNR 6852-1 adhesive. Some details that are expected when comparing a numerical simulation with an experimental curve are evident: experimental curves usually fail at a displacement lower than the numerical one (due to the inherent microscopic flaws of real world materials), and also the stiffness of the elastic zone of the curve is usually higher in the numerical results (due to numerical modelling considering a perfectly rigid displacement inducement method, which does not happen in reality as test machines have various mechanical components that slightly elastically deform, and is also attributed to the fact that the followed experimental procedure includes a pre-cracking stage, performed according to ISO 25217, which induces a change in the initial crack length of the specimens and influences the initial stiffness of the curve), which is especially visible in the case of SikaPower 4720 but is also present in the case of Nagase XNR 6852-1. Regarding the effectiveness of each type of CZM, the trapezoidal CZM appears to be the best in matching the experimental maximum load for both cases, while both the triangular and exponential CZM result in a slightly lower experimental maximum load. This occurs because ductile adhesives are normally better modelled using a 
trapezoidal CZM, which due to its plateau (see Figure1 1 (c) for the case of $t_{m_{1}}=t_{m_{2}}$ and Figure 7 zone 3) is more appropriate to model the gradual degradation of the adhesive layer of the studied adhesives.

Regarding the tests with moisture degradation, Equation 7 is used together with the values from Table 2 by the element to estimate the degraded CZM properties, such that each type of curve is adapted automatically in the beginning of the simulation to match the degraded stage being modelled. Figure 12 presents the comparison between simulation results and the experimental maximum load, $P_{\max }$, together with the experimental failure displacement, $d_{\max }$, as a function of the adhesive and ageing stage, which are shown in Table 3 .

Table 3: Definition of each aged stage for both studied adhesives.

\begin{tabular}{l|cc}
\hline & Nagase XNR 6852-1 & SikaPower 4720 \\
\hline $\mathrm{RH}=0 \%$ & Case A1 & Case B1 \\
$\mathrm{RH}=25 \%$ & Case A2 & Case B2 \\
$\mathrm{RH}=40 \%$ & Case A3 & Case B3 \\
$\mathrm{RH}=60 \%$ & Case A4 & Case B4 \\
\hline
\end{tabular}

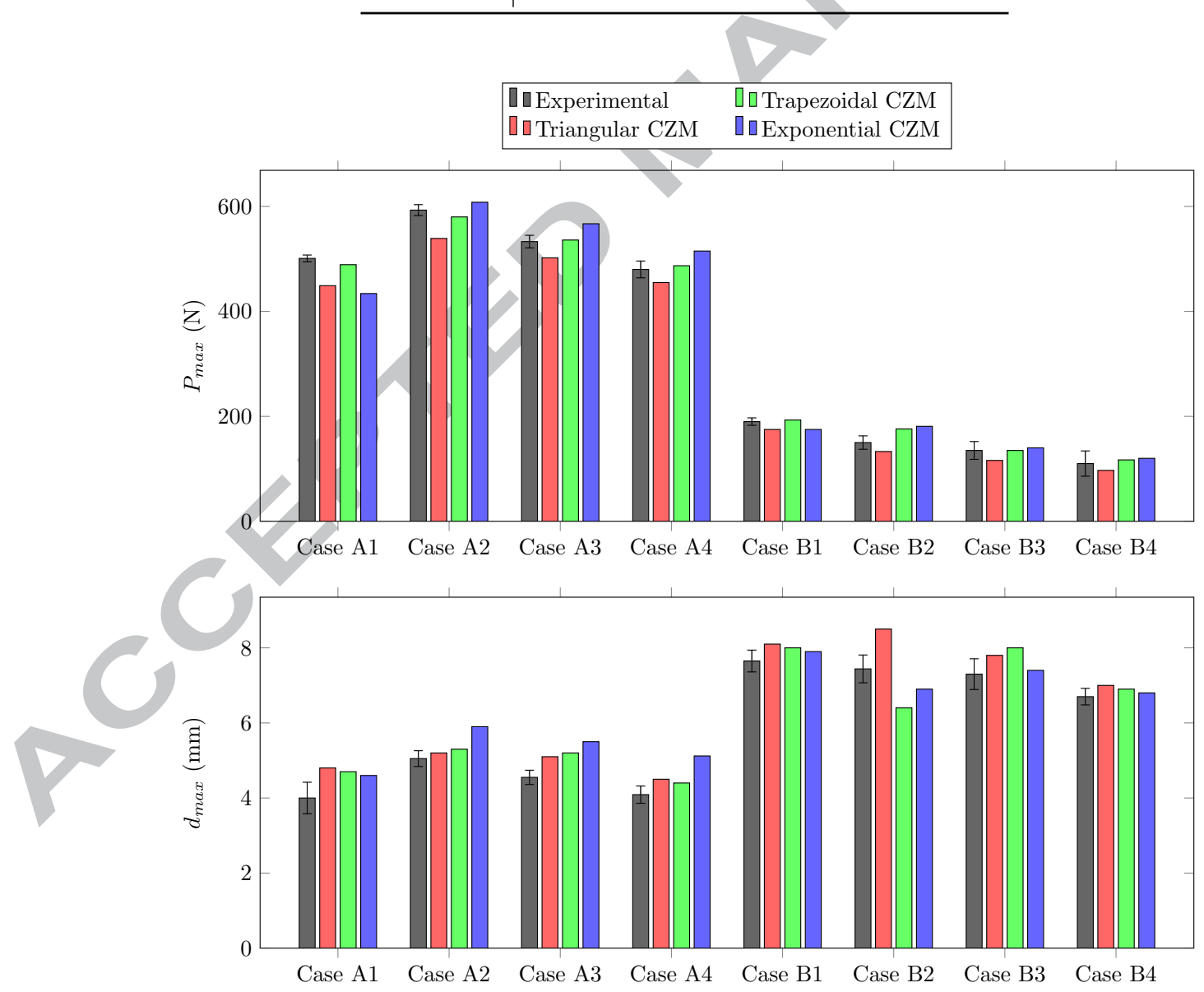

Figure 12: Comparison between experimental and numerical values of the maximum load and displacement for both adhesives in each aged state. 
The Nagase XNR 6852-1 results generally present a higher maximum load than SikaPower 4720, which happens because Nagase XNR 6852-1 has higher mechanical properties and also because the substrate thickness is higher (therefore allowing for less bending in the elastic stage). The maximum displacement is also generally lower for the Nagase XNR 6852-1 adhesive because of the same reason (by bending less, the thicker substrate will force separation for a lower value of displacement, thus breaking the joint sooner). Regarding the ageing stages, it is visible that the maximum load for Nagase XNR 6852-1 increases from the unaged stage $(\mathrm{RH}=0 \%)$ to $25 \%$ relative humidity, which matches the experimental measurements for those two stages [23], and after $\mathrm{RH}=25 \%$ there is a gradual decrease of the maximum load with an increase in relative humidity. For SikaPower 4720 there is a gradual decrease of the maximum load for all consecutive stages after the unaged stage. As for the results of the numerical simulation for the three types of studied traction-separation laws, the numerical results match the experimental curves in all cases with varying degrees of accuracy, which fluctuates due to the type of CZM being more or less appropriate for the situation. Generally, the trapezoidal $\mathrm{CZM}$ is closer to the experimental value than the other two options, but exceptions exist, although in all cases the approximation for any case is acceptably near the experimental values.

\subsection{Fatigue tests}

\subsubsection{Crack growth simulation}

Crack growth is controlled during the numerical simulation by applying Equation 8, which will degrade the traction-separation law as to induce crack propagation as pictured in Figure 5. This will lead to propagation of the crack in an otherwise static simulation, and by adjusting the $K$ constant one can influence the crack propagation behaviour and, specifically, the crack length increases as a function of the number of applied cycles. Figure 13 shows the crack propagation plots (Paris Law) for three different $K$ values for a DCB joint.
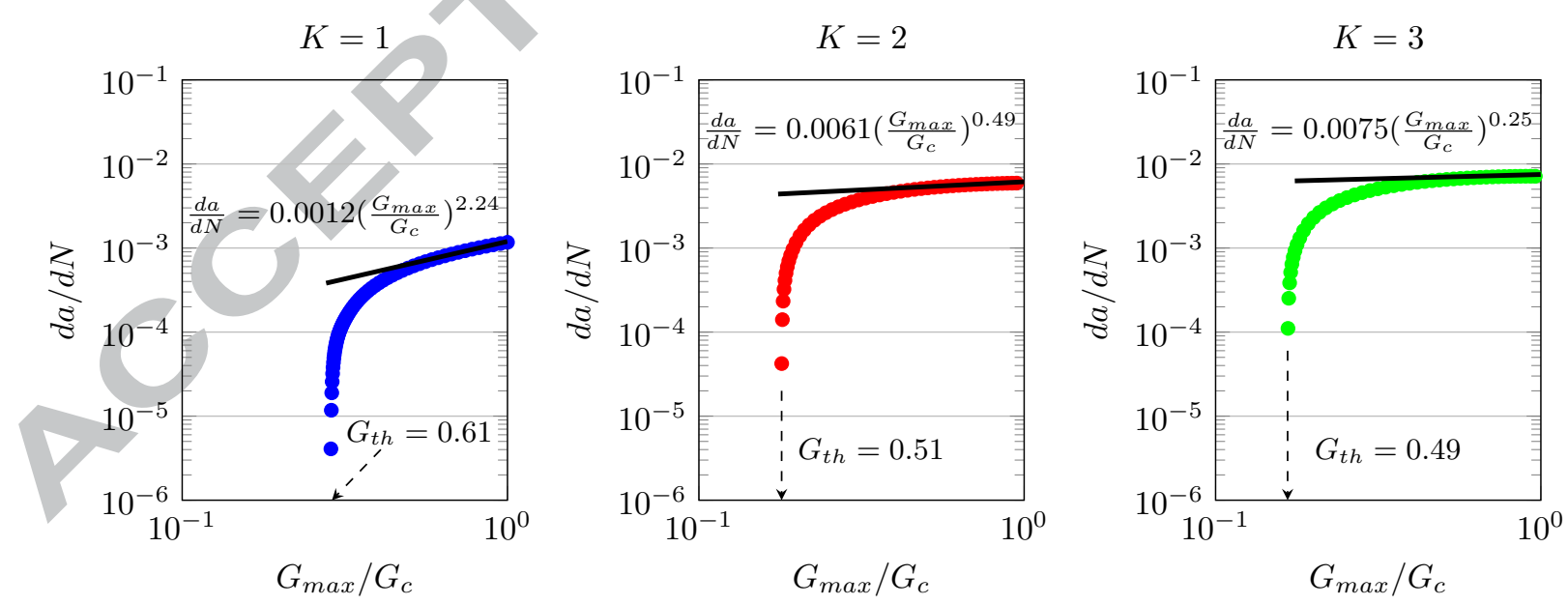

Figure 13: Numerical crack propagation curves for three cases: $K=1, K=2$ and $K=3$.

Altering the value of $K$ has a distinct influence in the Paris Law constants, as an increase in $K$ translates to a decrease in the Paris Law slope, a decrease in $G_{t h}$ and an increase in the $y$-axis intersection value. This 
makes sense because an increase in $K$ will lead to a quicker decrease in CZM parameters (see Figure 5 (b)), therefore decreasing them faster will result in the crack initiating sooner, thus lowering the value for the crack initiation threshold $G_{t h}$. Furthermore, after the quicker decrease in CZM parameters in the earlier cycles, the parameters will stabilize (gradually halting the decrease), which translates to the crack propagating much more gradually and slower, thus decreasing the slope of the corresponding Paris Law. It is finally concluded that adjusting the $K$ constant has a noticeable influence in the Paris Law parameters, thus $K$ can be chosen as a function of experimental results to closely match the experimental Paris Law curves using the proposed element.

\subsubsection{Prediction of failure cycles}

Prediction of the number of cycles is based on Equation 9 , where an average $d a / d N$ value is selected as a function of the experimental Paris Law results for the specific condition [25], with Figure 14 presenting the comparison between experimental and numerical results for the same cases shown in Table 3 A logarithmic representation was chosen as it is the usual procedure for studying fatigue life (for example, in S-N curves).

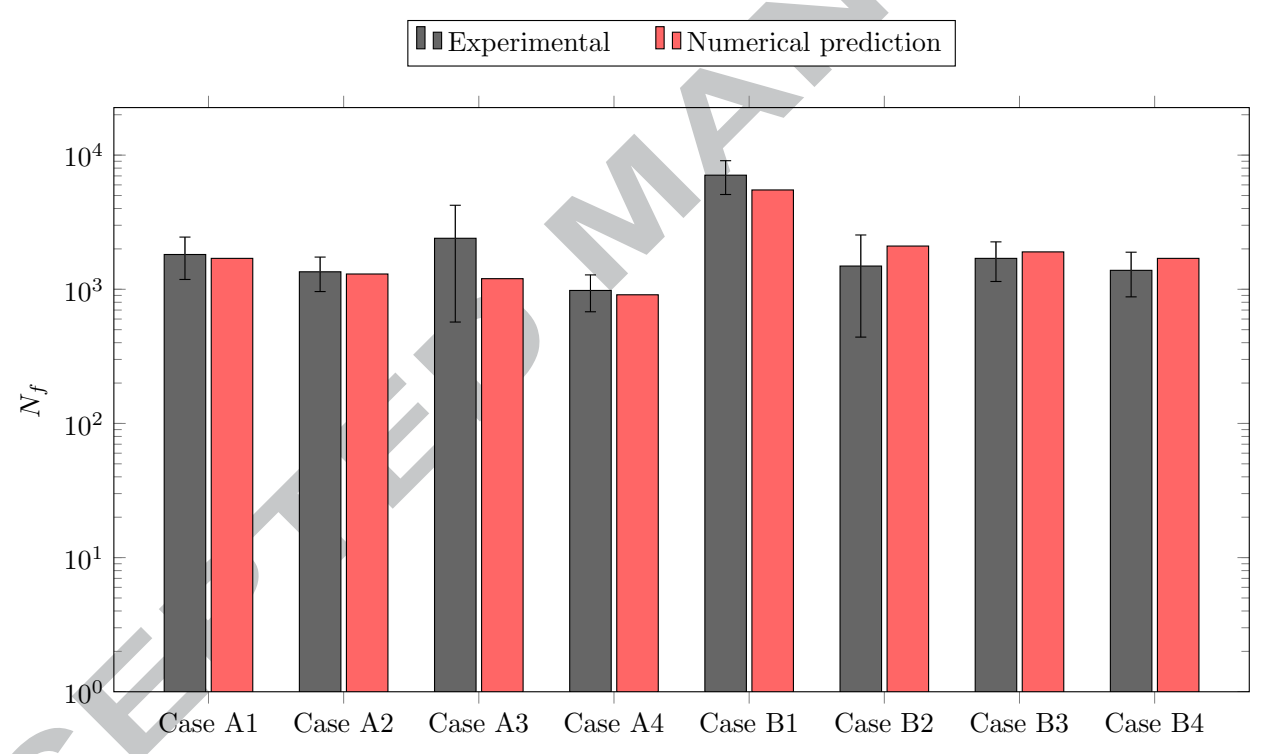

Figure 14: Comparison between experimental and numerical prediction for the number of cycles at failure for each of the studied adhesives and ageing conditions.

It is visible that for almost all cases the predicted number of cycles by the proposed approach is very near the experimental cycles and well within the standard deviation due to experimental scatter, validating their accuracy. Figure 14 presents a comparison only for the low cycle fatigue regime as fatigue testing has been performed, primarily with the intent of obtaining crack growth curves, for $60 \%$ of the static failure load [25], leading to failure cycles in the range of $10^{3}-10^{4}$. Due to the straightforward nature of Equation 9, the high cycle fatigue regime can also be predicted, although future work for lower load percentages of the static failure load will confirm this. 


\section{Conclusions}

A finite element based on the cohesive zone model formulation is proposed. The two main advantages of the element are the ability to model joints degraded by humidity, using degradation formulas based on experimental data that adapt the traction-separation law, and joints subjected to fatigue, using proposed relationships that both damage the bonded area gradually (inducing fatigue crack propagation within the numerical analysis) and predict the number of cycles at failure for the bonded joint.

It is shown that the degradation relationships allow for numerical results in both static and fatigue conditions very close to the experimental tests. Additional advantages of this finite element include the implementation of several types of traction-separation laws, such as triangular with exponential softening, trapezoidal, exponential and the possibility to implement a fully customized CZM law. It is shown that the trapezoidal CZM is the one that results in curves and values closer to the experimental tests.

Lastly, a MATLAB interface was developed which controls mesh generation for the requested geometry, creation of the input simulation files for $\mathrm{ABAQUS}^{\circledR}$, monitoring of the simulation while running, and finally extraction and visualization of results, which has proved to be a useful tool in speeding up the numerical analysis process from start to finish.

It is evident that the element shown here presents several advantages over conventional solutions, and will certainly be a valuable tool for any engineer modelling joints subjected to degradation and fatigue, as well as joints that have specific requirements concerning the type of traction-separation law best fit for the situation.

This work is a starting point to a fully inclusive methodology that should include additional loading modes (mode II, mode III, mixed-mode) as well as further fatigue considerations (compressive and alternating loads).

\section{Acknowledgements}

The authors would like to thank Fundação para a Ciência e a Tecnologia (FCT) for financing this work through grant EXCL/EMS-PRO/0084/2012, Science and Technology for Competitive and Sustainable Industries (SciTech) through grant NORTE-01-0145-FEDER-000022, Nagase Chemtex for suplying the Nagase XNR 6852-1 adhesive, and Sika for supplying the SikaPower 4720 adhesive.

\section{References}

[1] L. F. M. da Silva, A. Öchsner, R. D. Adams, Handbook of adhesion technology, Springer Science \& Business Media, 2011.

[2] L. F. M. da Silva, D. A. Dillard, B. Blackman, R. D. Adams, Testing adhesive joints: best practices, John Wiley \& Sons, 2012.

[3] L. F. M. da Silva, R. D. Campilho, Advances in numerical modelling of adhesive joints, in: Advances in Numerical Modeling of Adhesive Joints, Springer, 2012, pp. 1-93. 
[4] G. I. Barenblatt, The mathematical theory of equilibrium cracks in brittle fracture, Advances in applied mechanics 7 (1962) 55-129.

[5] A. Hillerborg, M. Modéer, P.-E. Petersson, Analysis of crack formation and crack growth in concrete by means of fracture mechanics and finite elements, Cement and concrete research 6 (6) (1976) 773-781.

[6] R. D. S. G. Campilho, M. Costa, G. Viana, L. F. M. da Silva, Parameter identification in cohesive zone modelling, Strength Prediction of Adhesively-Bonded Joints (2017) 161.

[7] C. C. R. G. de Sousa, R. D. S. G. Campilho, E. A. S. Marques, M. Costa, L. F. M. da Silva, Overview of different strength prediction techniques for single-lap bonded joints, Proceedings of the Institution of Mechanical Engineers, Part L: Journal of Materials: Design and Applications 231 (1-2) (2017) 210-223.

[8] K. Park, G. H. Paulino, Computational implementation of the ppr potential-based cohesive model in abaqus: educational perspective, Engineering fracture mechanics 93 (2012) 239-262.

[9] R. D. S. G. Campilho, M. D. Banea, J. A. B. P. Neto, L. F. M. da Silva, Modelling adhesive joints with cohesive zone models: effect of the cohesive law shape of the adhesive layer, International Journal of Adhesion and Adhesives 44 (2013) 48-56.

[10] C. D. M. Liljedahl, A. D. Crocombe, M. A. Wahab, I. A. Ashcroft, Modelling the environmental degradation of the interface in adhesively bonded joints using a cohesive zone approach, The Journal of Adhesion 82 (11) (2006) 1061-1089.

[11] K. B. Katnam, J. P. Sargent, A. D. Crocombe, H. Khoramishad, I. A. Ashcroft, Characterisation of moisture-dependent cohesive zone properties for adhesively bonded joints, Engineering Fracture Mechanics 77 (16) (2010) 3105-3119.

[12] A. Mubashar, I. A. Ashcroft, G. W. Critchlow, A. D. Crocombe, Modelling cyclic moisture uptake in an epoxy adhesive, The Journal of Adhesion 85 (10) (2009) 711-735.

[13] M. F. S. F. de Moura, J. P. M. Gonçalves, Cohesive zone model for high-cycle fatigue of adhesively bonded joints under mode i loading, International Journal of Solids and Structures 51 (5) (2014) 11231131.

[14] F. Moroni, A. Pirondi, A procedure for the simulation of fatigue crack growth in adhesively bonded joints based on the cohesive zone model and different mixed-mode propagation criteria, Engineering Fracture Mechanics 78 (8) (2011) 1808-1816.

[15] H. Khoramishad, A. D. Crocombe, K. B. Katnam, I. A. Ashcroft, Predicting fatigue damage in adhesively bonded joints using a cohesive zone model, International Journal of fatigue 32 (7) (2010) 1146-1158.

[16] A. Turon, J. Costa, P. P. Camanho, C. G. Dávila, Simulation of delamination in composites under high-cycle fatigue, Composites Part A: applied science and manufacturing 38 (11) (2007) 2270-2282. 
[17] O. Nguyen, E. A. Repetto, M. Ortiz, R. A. Radovitzky, A cohesive model of fatigue crack growth, International Journal of Fracture 110 (4) (2001) 351-369.

[18] K. L. Roe, T. H. Siegmund, An irreversible cohesive zone model for interface fatigue crack growth simulation, Engineering fracture mechanics 70 (2) (2003) 209-232.

[19] O. Zienkiewicz, R. Taylor, The Finite Element Method: The basis, Fluid Dynamics, ButterworthHeinemann, 2000.

[20] D. Systèmes, ABAQUS 6.13-4 Documentation, Providence, RI, USA (2013).

[21] B. F. Sørensen, T. K. Jacobsen, Determination of cohesive laws by the j integral approach, Engineering fracture mechanics 70 (14) (2003) 1841-1858.

[22] W. K. Loh, A. D. Crocombe, M. M. A. Wahab, I. A. Ashcroft, Modelling anomalous moisture uptake, swelling and thermal characteristics of a rubber toughened epoxy adhesive, International journal of adhesion and adhesives 25 (1) (2005) 1-12.

[23] M. Costa, G. Viana, L. F. M. da Silva, R. D. S. G. Campilho, Effect of humidity on the mechanical properties of adhesively bonded aluminium joints, Proceedings of the Institution of Mechanical Engineers, Part L: Journal of Materials: Design and Applications (2016) 1464420716645263.

[24] M. Costa, G. Viana, L. F. M. da Silva, R. D. S. G. Campilho, Environmental effect on the fatigue degradation of adhesive joints: a review, The Journal of Adhesion 93 (1-2) (2017) 127-146.

[25] M. Costa, G. Viana, L. F. M. da Silva, R. D. S. G. Campilho, Effect of humidity on the fatigue behaviour of adhesively bonded aluminium joints, Latin American Journal of Solids and Structures 14 (1) (2017) 174-187.

[26] M. Costa, G. Viana, C. Canto, L. F. M. da Silva, M. D. Banea, F. Chaves, R. D. S. G. Campilho, A. A. Fernandes, Effect of the size reduction on the bulk tensile and double cantilever beam specimens used in cohesive zone models, Proceedings of the Institution of Mechanical Engineers, Part L: Journal of Materials: Design and Applications 230 (5) (2016) 968-982. 